\title{
Frequency-Domain Identification of Linear Time-Periodic Systems using LTI Techniques
}

\author{
Matthew S. Allen \\ Assistant Professor \\ Department of Engineering Physics \\ University of Wisconsin-Madison \\ msallen@engr.wisc.edu
}

\begin{abstract}
\end{abstract}
A variety of systems can be faithfully modeled as linear with coefficients that vary periodically with time or Linear Time-Periodic (LTP). Examples include anisotropic rotor-bearing systems, wind turbines and nonlinear systems linearized about a periodic trajectory; all of these have been treated analytically in the literature. However, few methods exist for experimentally characterizing LTP systems. This paper presents a set of tools that can be used to experimentally characterize an LTP system, using a frequency domain approach and utilizing existing algorithms to perform parameter identification. One of the approaches is based on lifting the response to obtain an equivalent Linear Time-Invariant (LTI) form and the other based on Fourier series expansion. The development focuses on the pre-processing steps needed to apply LTI identification to the measurements, the post-processing needed to reconstruct the LTP model from the identification results and the interpretation of the measurements. This approach elucidates the similarities between LTP and LTI identification, allowing the experimentalist to transfer insight from time-invariant systems to the LTP identification problem. The approach determines the model order of the system, and post processing reveals the shapes of the time-periodic functions comprising the LTP model. Further post-processing is also presented that allows one to generate the full state transition matrix and the time-varying state matrix of the system from the parametric model if the measurement set is adequate. The experimental techniques are demonstrated on simulated measurements from a Jeffcott rotor mounted on an anisotropic, flexible shaft, supported by anisotropic bearings.

\section{INTRODUCTION}

Many important dynamic systems can be modeled as Linear Time-Periodic (LTP). When a system has periodically varying parameters, it is exceedingly important to discover and accurately model this character since it can lead to parametric resonances which are not present for a Linear TimeInvariant (LTI) approximation to the system. Floquet initiated the study of LTP systems in the 1800's [1], and modern Floquet theory has been applied to a variety of mechanical systems such as helicopters [2], wind turbines or other bladed machines [3], [4], mechanisms [5], buckling problems [3], rotating machinery [6-9] and many others [10-12]. Linear time-periodic system models are also frequently encountered in analysis of nonlinear systems, as it often proves beneficial to linearize the nonlinear system about a periodic trajectory to study its stability.

Much progress has been made in the past twenty years towards analysis and control of linear and nonlinear time-periodic systems, as many concepts for LTI systems are readily extended to LTP systems $[5,13]$. On the other hand, experimental techniques are well developed for LTI systems, but LTP system identification has received relatively little attention. Experimental methods are needed in a number of applications, for example when it is impossible to determine the parameters or periodic functions comprising the LTP model for a system from first principles. They may also be useful to monitor the health of a system [14], to validate analytical models and to diagnose modeling errors.

Many texts cover system identification approaches for general time-varying systems, but these either require a large ensemble of synchronized data or else are not appropriate if the system's parameters vary quickly with time [15]. Two early works that overcome these limitations are those of Hench [16] and Verhaegen and Yu [17]. Both presented subspace methods for LTP systems. The latter was recently extended by Felici et al. so that one can extract the state sequence with a consistent basis [18]. Liu presented a similar method in [19] that is applicable to LTP and to arbitrarily linear time-varying 
(LTV) systems, and applied his method to simulated measurements from a robot arm linearized about a periodic trajectory. Shi, Law and Li recently expanded upon that work applying the algorithm to simulated measurements from various systems [20]. Peters and Wang [21] independently arrived at related methodology that sought to more accurately and efficiently generate the state transition matrix of an analytical LTP system [21] and to allow one to characterize the effects of hidden aerodynamic states [22]. They subsequently applied their technique to find the Floquet multipliers of a laboratory helicopter rotor $[23,24]$. All of these are time-domain methods and focus on the discrete-time representation for an LTP system. The advantages and limitations of subspace methods for LTI systems are fairly well established now [25, 26], and in many instances frequency domain approaches are preferred [27-29]. Furthermore, none of these works proposes how one may recover the continuous-time system matrices from the discrete-time model that is identified.

Werely and Hall developed the concept of harmonic transfer functions (HTF) to treat LTP systems in the frequency domain [30]. Siddiqui further developed these ideas in his thesis [31] and applied them to synthesized data. The methods were also recently applied to measurements from a wind tunnel model of a helicopter rotor [32], although no meaningful time-periodic behavior could be detected in those measurements. Van Landuit et al. [33] independently developed an elegant algorithm that shares many of the elements of their approach; Either approach tends to require long time records and carefully controlled inputs [33]. Of the works cited thus far, only those in [24], [32] and [33] have employed actual experimental measurements from mechanical systems. A number of other methods also exist that are applicable to both nonlinear and LTP systems, although most are limited to single-degree of freedom or low order systems, such as the restoring force method [34, 35], the Hilbert transform, and a few others [36-38].

This work presents a frequency-domain approach that allows one to characterize an LTP system using the same methodology, algorithms and intuition that are used for LTI systems. The focus is on developing the pre- and post-processing needed so that any suitable LTI identification routine can be used to process free response measurements from an LTP system, rather than develop a new algorithm that is specific to LTP identification. Particular emphasis is placed on elucidating the differences between the LTP response and that of an LTI system because that understanding is critical to finding the proper system order and to correctly distinguishing time-periodic behavior from noise, anomalies due to the transducers or instrumentation, signal processing errors, and other factors that are common to experimental measurements. One of the advantages of this approach is that it allows experimentalists to transfer insight from LTI identification to the LTP problem. Assuming that the fundamental period of the system is known, a lifting scheme [17, 39] is used to transform the measurements into a set of responses that can be described by an LTI model. A Fourier series description is also developed that can, in principle, be used to address the case where the fundamental period is not known. The Fourier series description used here is the converse of the Harmonic Transfer Function approach developed in [30-32]. Either approach identifies a parametric model for the modal parameters of the STM, which can be used to characterize the system [40]. This work also shows how one can estimate the STM and the time-varying state matrix of the system if the measurements are of sufficient quality.

One important feature of this approach is that it does not require an initial guess regarding the system's model order nor restrictive assumptions about its form. The model order is identified by interrogating the response data, as is done for LTI systems, and nothing is assumed about the model form except that it is time-periodic. Aspects of the methods presented here were developed by the author in a few preliminary works $[41,42]$. They have recently been applied to identify LTI structural modes from continuous-scan laser vibrometer measurements $[43,44]$, where the LTP response has many modes with complicated time-periodic patterns, so the aforementioned features have proved highly beneficial.

This paper is organized as follows. Section 2 reviews Floquet theory and presents two strategies for identifying the parameters of LTP systems from free response data. Methods for finding the state transition matrix and the system matrix from the identified models are then presented. These methods are applied to an analytical system whose parameters vary with time in Section 3, which consists of a simple model of a Jeffcott rotor on an anisotropic shaft and supported by anisotropic bearings. Section 4 presents some conclusions.

\section{Theoretical Development}

The following section briefly reviews some pertinent concepts from Floquet Theory. Section 2.2 presents two descriptions that can be used to identify parametric models of LTP systems using LTI 
methods, and Section 2.3 describes how to reconstruct the LTP state transition matrix and state matrix from the parametric model.

\subsection{Review of Floquet Theory for LTP Systems} form

The equations governing a linear time-varying system may be written in the following state space

$$
\begin{aligned}
& \dot{x}=A(t) x \\
& y=C(t) x
\end{aligned}
$$

where $x$ is the $N \times 1$ system state vector, $y$ the $N_{\mathrm{o}} \times 1$ vector of outputs and the matrices $A(t)$ and $C(t)$ are periodic with period $T_{A}$ so that $A\left(t+T_{A}\right)=A(t)$ and $C\left(t+T_{A}\right)=C(t)$. The state transition matrix can be used to transfer the state vector from the initial state $x\left(t_{0}\right)$ at time $t_{0}$ to the state at time $t$ as follows

$$
x(t)=\Phi\left(t, t_{0}\right) x\left(t_{0}\right)
$$

Floquet's theorem [45] states that if the state transition matrix of an LTP system with period $T_{A}$ is diagonalizable, then it can be decomposed as follows,

$$
\Phi\left(t, t_{0}\right)=\Psi(t) \exp \left(\Lambda_{R}\left(t-t_{0}\right)\right) \Psi\left(t_{0}\right)^{-1}
$$

where $\Psi(t)=\left[\left\{\psi_{1}\right\}, \ldots,\left\{\psi_{\mathrm{N}}\right\}\right]$ is the time varying modal matrix of the STM, $N$ denotes the number of modes and $\Lambda_{R}$ is a diagonal matrix containing the Floquet exponents of the system. The Floquet multipliers $\mu_{r}$ are related to the Floquet exponents $\lambda_{r}$ by $\mu_{r}=\exp \left(\lambda_{r} T_{A}\right)$. The Floquet exponents can be determined from the Floquet multipliers only up to a constant,

$$
\lambda_{r}=\frac{\ln \left(\mu_{r}\right)}{T_{A}}+i n \omega_{A}
$$

where $\omega_{A}=2 \pi / T_{A}$ and $n$ is an arbitrary integer. This is of little consequence because one can correctly reconstruct the STM in eq. (3) with any $n$, and likewise all of the following is valid for any $n$. However, as the strength of the systems time periodicity decreases, the mode vectors $\left\{\psi_{r}(t)\right\}$ reduce to constant vectors only for a specific $n$.

Equation (3) can be expressed in summation form as follows

$$
\begin{aligned}
& \Phi\left(t, t_{0}\right)=\sum_{r=1}^{N} R_{\Phi}(t)_{r} \exp \left(\lambda_{r}\left(t-t_{0}\right)\right), \\
& R_{\Phi}(t)_{r}=\left\{\psi_{r}(t)\right\}\left\{L_{r}\left(t_{0}\right)\right\}^{T}
\end{aligned},
$$

where $\left\{L_{r}\left(t_{0}\right)\right\}^{T}$ is the $r$ th row of $\Psi\left(t_{0}\right)^{-1}$. The residue matrices $R_{\Phi}(t)_{r}$ are the product of a periodic column vector and a periodic (or constant) row vector, and hence are periodic and rank one. The model for an observed response obtained by combining eq. (1), (2) and (5) resulting in

$$
\begin{aligned}
& y(t)=\sum_{r=1}^{N} R_{Y}(t)_{r} \exp \left(\lambda_{r}\left(t-t_{0}\right)\right) \\
& R_{Y}(t)_{r}=C(t)\left\{\psi_{r}(t)\right\}\left\{L_{r}\left(t_{0}\right)\right\}^{T}\left\{x_{0}\right\}
\end{aligned},
$$

where $R_{Y}(t)_{r}$ is the residue of the observed response.

\subsection{Identifying Parametric Models for LTP Systems}

The following subsections show how the free response of an LTP system can be exactly parameterized as an LTI system using two different methods. The pre- and post-processing necessary to extract the parameters of the LTP system from the output of an LTI system identification routine are presented, and important issues regarding the selection of the LTI identification routine are enumerated. 


\subsubsection{Lifting Approach}

Consider the response in eq. (5), sampled at instants $t_{n}=t_{0}+n \Delta t$ for $n=0 \ldots N_{s}-1$ and with $\Delta t=$ $T_{A} / P$, so the response is sampled an integer number $P$ times per fundamental period and an integer number of cycles $N_{c}=N_{s} / P$ are collected. The periodicity of $R_{Y}(t)_{r}$ effectively vanishes if one always samples at the same point within the fundamental period, so the sampled response of the system to a single initial condition is exactly represented as a superposition of exponentials with constant amplitudes. This idea has been used in a few previous works $[18,38,46]$, where it was called "lifting." The lifted response is

$$
y^{L}=\left[y_{0}{ }^{T}, y_{1}^{T}, \cdots, y_{P-1}{ }^{T}\right]^{T},
$$

where $y_{k}$ is the response at the time instants $t_{n}$ for $n=k+m P$ for $k=0 \ldots P-1$ and $m=0 \ldots N_{c}-1$.

The free response of the lifted system has the following form

$$
y^{L}(t)=\sum_{r=1}^{N} R_{r}^{L d} \exp \left(\lambda_{r} m T_{A}\right) \quad m=0 \ldots N_{c}-1,
$$

where $R^{L d}{ }_{r}$ is related to $R_{k, r}$ in the same way that $y^{L}$ is related to $y_{k}$, and

$$
R_{k, r}=R_{Y}\left(t_{k}\right)_{r} \exp \left(\lambda_{r}\left(t_{k}-t_{0}\right)\right) \quad k=0 \ldots P-1 .
$$

The exponential term is important because it accounts for the delay (hence the $d$ in the notation $R^{L d}{ }_{r}$ ) between $t_{0}$ and the beginning of each response $y_{k}$. For convenience, we also define $R_{r}^{L}$ which is identical to $R^{L d}{ }_{r}$ but without the exponential term, which is the lifted form of $R_{Y}(t)_{r}$. Equation (8) has the same form as the response of an LTI system, so LTI parameter identification routines can be applied either to $y^{L}(t)$, to a discrete time representation of eq. (8), or to its frequency domain dual

$$
Y^{L}(\omega)=\sum_{r=1}^{N} \frac{R_{r}^{L d}}{i \omega-\lambda_{r}},
$$

which also has the standard form for an LTI system [47]. In either case, an LTI algorithm will extract the residue vectors $R^{L d}{ }_{r}$ and Floquet Exponents from the responses.

By analogy with LTI systems, measurements from $N_{i}$ different initial conditions can be considered to form an $N_{\mathrm{o}} \times N_{i}$ matrix of responses at each frequency, in which case it follows from the definition of $R^{L d}{ }_{r}$ that the resulting residue matrices are rank one, each column being proportional to the mode vectors $C\left(t_{k}\right)\left\{\psi_{r}\left(t_{k}\right)\right\}$, just as for an LTI system. Hence, concepts such as the Mode Indicator Function [47, 48] and MIMO identification [49], which are important tools for detecting close natural frequencies, are directly applicable. If $P$ is large, then there will be a large number of outputs and one should seek an algorithm that can efficiently handle that case, such as the Least Squares Complex Frequency Domain Algorithm (LSCF) [50] or the Algorithm of Mode Isolation (AMI) [51-54]. One important feature of all of these approaches is that they consider all of the responses simultaneously to obtain the best global estimate of the Floquet exponents.

One important difference between identification of the lifted LTP system and standard LTI identification is that the bandwidth of $Y^{L}(\omega)$ is limited to half the fundamental frequency $\omega_{A}$, which may alias the lifted response. In that case, one can account for the aliasing using the sampled (star *) Laplace transformation [39,55], which is equivalent to fitting the response to a model with Z-domain polynomials [49], which have the same form as eq. (10) but with $i \omega$ replaced with $z=\exp \left(i \omega T_{A}\right)$. However, the distinction is usually not important for lightly damped structures if the Floquet exponents are not too close to 0 or $\omega_{\mathrm{A}} / 2$.

Whatever approach is used, the modal parameter identification routine returns an estimate for $\lambda_{r}$ and the rank one residue matrices $R^{L d}{ }_{r}$. One can then estimate $R_{r}^{L}$ by multiplying each block of $R^{L d}{ }_{r}$ by $\exp \left(-\lambda_{r}\left(\mathrm{t}_{\mathrm{k}}-\mathrm{t}_{0}\right)\right)$. Note that this multiplication involves the identified Floquet exponents, whose imaginary part contains an arbitrary integer multiple of $\omega_{A}$. Hence, the estimated $R_{r}^{L}$ should always be periodic, but the Fourier series expansion of $R_{r}^{L}$ may be shifted by an arbitrary integer. This is important only when interpreting $R_{r}^{L}$ because the arbitrary integer vanishes when combining $R_{r}^{L}$ and $\lambda_{r}$ to form the STM. 


\subsubsection{Fourier Series Expansion (FSE) Method}

Because the residues $R_{Y}(t)_{r}$ in eq. (5) are periodic, they can be readily expanded in a Fourier series. Here we shall presume that they can be adequately represented using a fixed number $\left(2 * N_{R}+1\right)$ of terms so that the response may be written as

$$
y(t)=\sum_{r=1}^{N}\left(\sum_{m=-N_{R}}^{N_{R}} B_{r, m} \exp \left(i m \omega_{A}\left(t-t_{0}\right)\right)\right) \exp \left(\lambda_{r}\left(t-t_{0}\right)\right)
$$

where $B_{r, m}$ is the $m$ th complex Fourier coefficient of the $r$ th residue. Factoring out the summations, the nature of the response is revealed.

$$
y(t)=\sum_{r=1}^{N} \sum_{m=-N_{R}}^{N_{R}} B_{r, m} \exp \left(\left(\lambda_{r}+i m \omega_{A}\right)\left(t-t_{0}\right)\right)
$$

This is mathematically equivalent to the impulse response of an LTI system with $N\left(2 N_{R}+1\right)$ eigenvalues $\lambda_{r}$ $+i m \omega_{A}$. The amplitude of each pseudo-mode's in the response is determined by the magnitude of the Fourier coefficient $B_{r, m}$ that defines its residue.

In light of eq. (12) we see that we can identify the parameters of an LTP system by processing its free response using an LTI system identification algorithm, without the lifting procedure. Equation (12) can then be used to interpret the result and/or reconstruct the LTP representation.

\subsubsection{Comparison and Discussion}

The most important distinction between the lifting and the FSE methods is that the former represents the system with many additional outputs while the latter is represented as a system with many modes (see eq. (12)). The lifting approach has significant advantages in this regard, because difficulty and the potential for ill-conditioning in most system identification (SysID) routines scales with the number of poles in the response, whereas a large number of response data sets are readily accommodated. Allemang and Brown [47] discuss this issue, describing system identification in terms of the matrix polynomials that are employed, the FSE method here corresponds to small matrix polynomials of high order while the lifting method corresponds to large matrix polynomials of low order. Furthermore, the frequency-domain responses obtained by the lifting method are easier to interpret - the system's model order is readily apparent.

Another disadvantage of the FSE method is that if standard LTI system identification routines are employed, then one cannot enforce the spacing between the eigenvalues that eq. (12) requires, specifically that each $\lambda_{r}$ is accompanied by multiples $\lambda_{r}+i m \omega_{A}$. This is an advantage if $\omega_{A}$ is not known, as one might be able to determine it from a collection of identified eigenvalues. On the other hand, in most applications $\omega_{A}$ can be measured and the estimated Floquet exponents most likely will not be consistent with the known $\omega_{A}$. One could perform a sort of averaging to obtain $\lambda_{r}$ and the corresponding integer $m$ for each exponent, but this process may be difficult and tedious if the system has many exponents or if $N_{R}$ is large $[43,44,56,57]$, especially if any of the contributions at $\lambda_{r}+i m \omega_{A}$ overlap. On the other hand, when the lifting method is employed, standard SysID algorithms can globally $[49,58]$ identify the Floquet exponents of the system utilizing all of the lifting responses simultaneously.

Despite these limitations, the FSE representation is useful in understanding the bandwidth required to acquire measurements and in assessing the fidelity of the measurements and of the resulting LTP model. Also, the FSE representation is needed to reconstruct the time-varying state matrix, as discussed in Sections 2.3. However, when the FSE representation is needed, the author has found it much more convenient to use the lifting method to identify a parametric model for the system from the measurements and then to expand the time varying residue matrices found by the lifting method into a Fourier series.

\subsection{Post-Processing}

The lifting and FSE methods provide a tool for identifying a response model for a system, but in some applications one needs to recover the state transition matrix or the time-varying state matrix of the LTP system as well. This section presents a series of post-processing operations that extract these from the pole-residue model obtained using the procedure outlined in Section 2.2. The STM and state matrix 
are most meaningful if the set of output points is sufficient to define a unique state, so the following assumes that this is the case. If the required conditions are not met, then one can apply the method used by Peters and Wang to augment the state vector with measurements from later time steps [21] (see also [17-19]), although the interpretation of the STM and state matrix are quite different in that case.

\subsubsection{Reconstructing the State Transition Matrix}

The following assumes that the measured outputs uniquely define the displacement state of the system through a known, full-rank, constant matrix $E$. Specifically, the state vector of interest is

$$
x_{d}(t)=E y(t)
$$

For example, one often has more output measurement locations than states, so an adequate state vector can be formed by choosing a subset of the outputs and $E$ is a Boolean matrix.

If the system is a second-order structural dynamic system, then one can use the derivatives of the displacement states to double the size of the state vector, so the final state vector is $x(t)=\left[x_{d}(t)^{\mathrm{T}}\right.$, $\left.x_{v}(t)^{\mathrm{T}}\right]^{\mathrm{T}}$ where $x_{v}(t)$ is the time-derivative of $x_{d}(t)$.

The methods in Section 2.2 identified a parametric model whose parameters (after applying the FFT to the lifted residues) are $\left\{B_{x}\right\}=E\left\{B_{y}\right\}$ and $\lambda_{r}$. Hence,

$$
x_{d}(t)=\sum_{r=1}^{2 N} \sum_{m=-N_{R}}^{N_{R}}\left\{B_{x}\right\}_{r, m} \exp \left(\left(\lambda_{r}+i m \omega_{A}\right)\left(t-t_{0}\right)\right)
$$

and one can differentiate to obtain the velocity states as follows.

$$
x_{v}(t)=\sum_{r=1}^{2 N} \sum_{m=-N_{R}}^{N_{R}}\left\{B_{x}\right\}_{r, m}\left(\lambda_{r}+i m \omega_{A}\right) \exp \left(\left(\lambda_{r}+i m \omega_{A}\right)\left(t-t_{0}\right)\right)
$$

One must assure that the identified response model is reliable, because the differentiation required could amplify errors in the components for large $m$. This approach is readily extended to the case where acceleration or velocity is measured in lieu of displacement.

One now has an FSE model for the state response $x(t)$ due to some initial condition $x\left(t_{0}\right)$, which is known by evaluating the FSE model at $t=t_{0}$. Hence, the only unknown in eq. (2) is the state matrix. However, there are $N^{2}$ unknowns in the state matrix at each time step and only one length $N$ response due to one length $N$ initial condition. Fortunately, one can generate the required responses by shifting each response by $n T_{A}$, where $n$ is an integer. Because the residues are periodic, one can use eqs. (14) and (15) to show that $x\left(t+n T_{A}\right)$ is equal to $x(t)$ with each term in the summation of the latter multiplied by $\exp \left(\lambda_{r} n T_{A}\right)$.

We now form the following system of linear equations.

$$
\left[\begin{array}{lll}
x(t) & \cdots & x\left(t+(N-1) T_{A}\right)
\end{array}\right]=\Phi\left(t, t_{0}\right)\left[\begin{array}{lll}
x\left(t_{0}\right) & \cdots & x\left(t_{0}+(N-1) T_{A}\right)
\end{array}\right]
$$

The matrix multiplying the STM on the right hand side is a constant matrix of initial conditions, which are known by evaluating eqs. (14) and (15) at $\underline{t}=\left[0, T_{A}, \ldots(N-1) T_{A}\right]$. That matrix is denoted $\left[x_{I C}\right]$, and after solving for the STM and after inserting eqs. (14) and (15) we obtain.

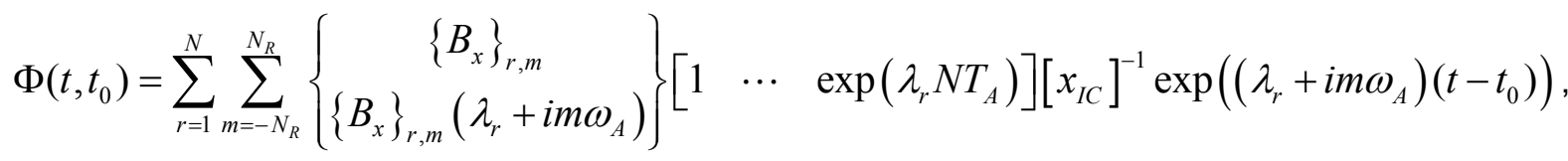

It is now apparent that one can take

$$
\left\{\psi_{r}(t)\right\}=\sum_{m=-N_{R}}^{N_{R}}\left\{\begin{array}{c}
\left\{B_{x}\right\}_{r, m} \\
\left\{B_{x}\right\}_{r, m}\left(\lambda_{r}+i m \omega_{A}\right)
\end{array}\right\} \exp \left(i m \omega_{A}\left(t-t_{0}\right)\right)
$$

and

$$
\left\{L_{r}\left(t_{0}\right)\right\}^{T}=\left[\begin{array}{lll}
1 & \cdots & \exp \left(\lambda_{r} N T_{A}\right)
\end{array}\right]\left[x_{I C}\right]^{-1},
$$


which completes the construction of the state transition matrix. All of the parameters of the STM in eq. (5) were identified from the parametric model provided by the methods in Section 2.2.

\subsubsection{Time-periodic State Matrix}

Once an FSE model for the State Transition Matrix has been identified, one can use the STM differential equation,

$$
\frac{d}{d t} \Phi\left(t, t_{0}\right)=A(t) \Phi\left(t, t_{0}\right)
$$

to solve for the time varying system matrix $A(t)$ as follows.

$$
A(t)=\left(\frac{d}{d t} \Phi\left(t, t_{0}\right)\right) \Phi\left(t, t_{0}\right)^{-1}
$$

In the applications that follow, this was accomplished numerically at each time step $t_{k}$ in the interval $\left[0, T_{A}\right)$. The derivative of the STM was found by differentiating eq. (17), as was done for the response model in eq. (15).

\section{Simulated Application}

The proposed system identification methods were evaluated by applying them to simulated data from an LTP system. The system represents a Jeffcott rotor on an anisotropic shaft, supported by anisotropic bearings, as illustrated in Figure 1. A lumped parameter model is also shown, consisting of a point mass suspended by two orthogonal, massless springs with spring constants $k_{R x}$ and $k_{R y}$. The springs are attached to a massless turntable that turns at constant speed $\Omega$. The turntable is fixed to ground by two massless springs $k_{F x}$ and $k_{F y}$. Defining non-dimensional time as $t=\omega_{n} t^{\prime}$ where $\omega_{n}=\sqrt{k_{F x} / m}$, the following non-dimensional equations of motion were obtained. The states are the $X$ and $Y$ coordinates in the fixed reference frame, and small displacements of the springs have been assumed.
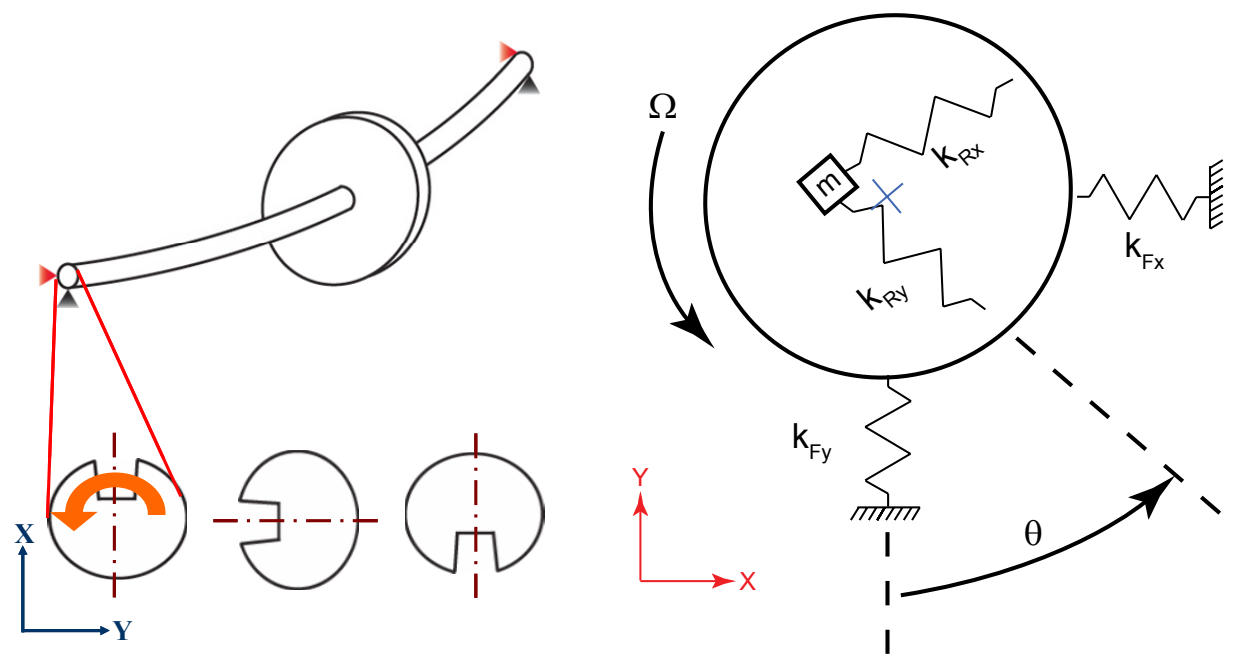

Figure 1: (left) Illustration of a rotor on an anisotropic shaft and anisotropic bearings. This system can be modeled as linear time-periodic. (right) Lumped parameter schematic of the linear timeperiodic system 


$$
\begin{aligned}
& {[I]\left\{\begin{array}{c}
\ddot{X} \\
\ddot{Y}
\end{array}\right\}+[\hat{C}]\left\{\begin{array}{c}
\dot{X} \\
\dot{Y}
\end{array}\right\}+[\hat{K}(t)]\left\{\begin{array}{c}
X \\
Y
\end{array}\right\}=\left\{\begin{array}{l}
0 \\
0
\end{array}\right\}} \\
& {[\hat{C}]=\omega_{n} c_{f}\left[\hat{K}_{F}\right],[R(t)]=\left[\begin{array}{cc}
\cos (\hat{\Omega} t) & \sin (\hat{\Omega} t) \\
-\sin (\hat{\Omega} t) & \cos (\hat{\Omega} t)
\end{array}\right], \hat{\Omega}=\frac{\Omega}{\omega_{n}}} \\
& {[\hat{K}(t)]=[R]\left[\hat{K}_{R}\right][R]^{T}\left([I]-\left([R]\left[\hat{K}_{R}\right][R]^{T}+\left[\hat{K}_{F}\right]\right)^{-1}[R]\left[\hat{K}_{R}\right][R]^{T}\right)} \\
& {\left[\hat{K}_{R}\right]=\left[\begin{array}{cc}
k_{R x} / k_{F x} \\
k_{R y} / k_{F x}
\end{array}\right],\left[\hat{K}_{F}\right]=\left[\begin{array}{ll}
1 & k_{F y} / k_{F x}
\end{array}\right]}
\end{aligned}
$$

The matrix $[I]$ denotes a two-dimensional identity matrix and $\Omega t$ is the turntable angle $\theta$. The timeperiodic stiffness matrix of the system depends on the ratios of the rotating and fixed spring stiffnesses to the stiffness $k_{F x}$ of the fixed spring in the x-direction. The factor $c_{f}$ controls the strength of the stiffness proportional damping in the system.

The following parameters are used in the following: $k_{R x} / k_{F x}=1, k_{R y} / k_{F x}=1.5, c_{f}=0.004, k_{F y} / k_{F x}=2$ and $\hat{\Omega}=0.5$. The equations of motion are periodic with a period $T_{A}=2 \pi$, which corresponds to one half of a revolution of the shaft. The natural frequencies of the system with the shaft held fixed at various angles ranged from 0.707 and $0.926 \mathrm{rad} / \mathrm{s}$. Stability analysis was performed using the method in [3], which reveals that the system is unstable for $0.72<\hat{\Omega}<0.755,0.785<\hat{\Omega}<0.825$ and $0.848<\hat{\Omega}<0.92$, so it would certainly be important to detect and properly characterize this system's time-periodicity if it is to operate at high speeds.

The response of the system to a unit impulsive force, administered at an angle of 45 degrees from the $x$ axis, was found using adaptive time integration, via Matlab's "ode45" function. The sample increment was chosen to be $\Delta t=0.126$, corresponding to 50 samples per half revolution of the shaft. The response was evaluated over a time window encompassing 512 revolutions of the shaft, which was adequate to allow the impulse response to decay to a small fraction of its initial amplitude.

Measurements were simulated of the displacements of the mass and of the turntable $(t t)$ in the fixed frame and in both directions, so the output vector is: $y=\left[X^{\mathrm{T}}, Y^{\mathrm{T}}, X_{t t}{ }^{\mathrm{T}}, Y_{t t}{ }^{\mathrm{T}}\right]^{\mathrm{T}}$. The turntable is massless, so its displacement is related to that of the mass by a time-varying transformation. This mimics the case where one might measure at multiple points on a flexible system, not knowing a priori how many points are required to capture the modes of the system. The response was contaminated with Gaussian white noise whose standard deviation was equal to $2 \%$ of the maximum response in each coordinate, to simulate a more realistic scenario. Also, the states corresponding to the velocities of the masses were not "measured"; only the four displacements just described were made available in the following.

\subsection{Response Model Identification}

Figure 2 shows the noise contaminated time response of the output $y_{1}=X$. An inset is also provided where markers highlight the response points at which the shaft (or turntable) is at either 0 or 180 degrees, which corresponds to the first lifted response $y_{1}{ }_{1}$. One can see that the lifted response aliases the true response frequency.

The discrete Fourier transform (DFT) of the outputs is shown in the upper panes of Figures 3 and 4, for $X$ and $Y$ and for $X_{t t}$ and $Y_{t t}$ respectively. The response in all of the outputs is dominated by two resonance peaks surrounding $0.8 \mathrm{rad} / \mathrm{s}$. Figure 3 shows two other pairs of peaks at 0.2 and $1.8 \mathrm{rad} / \mathrm{s}$, although the latter are almost completely masked by the noise. In contrast, Figure 4 shows noticeable harmonics only at $1.8 \mathrm{rad} / \mathrm{s}$. In any event, this two-degree of freedom LTP system appears to have at least six active modes in this impulse response, as explained by eq. (12). In that light, one might interpret the peaks at 0.2 and $1.8 \mathrm{rad} / \mathrm{s}$ as Fourier coefficients of the $0.8 \mathrm{rad} / \mathrm{s}$ modes, spaced from those by $\omega_{A}=$ 1.0 . 


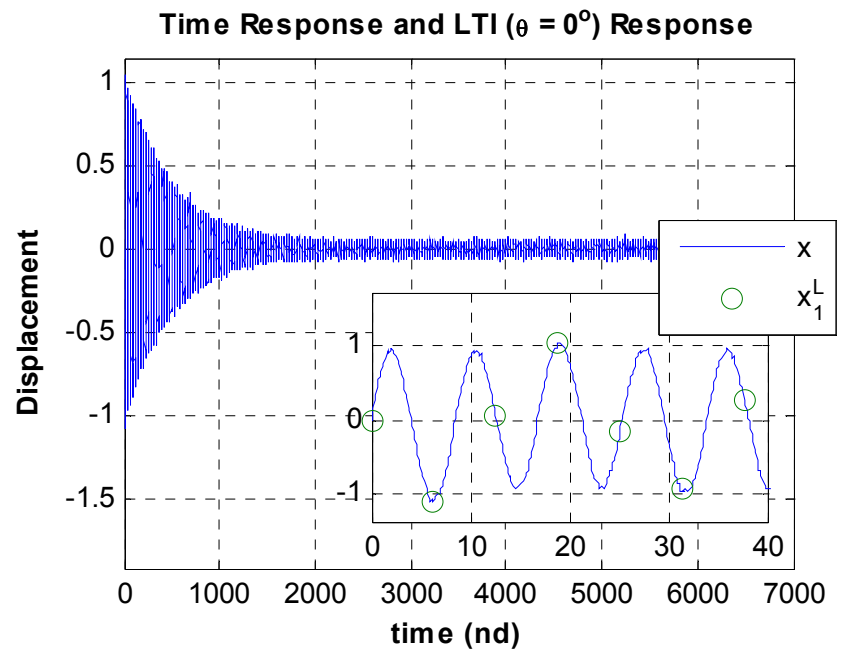

Figure 2: Noise contaminated time response of LTP system. Inset shows the response over the first few cycles. Circles mark the response points for which the shaft is at an angle of 0 or 180 degrees.

FFT of Response

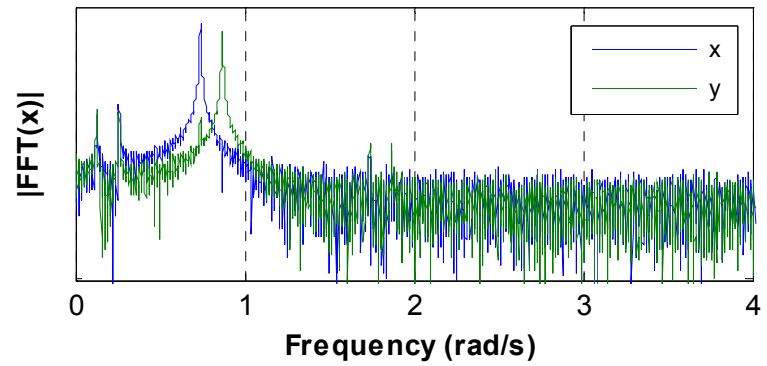

FFT of Lifted Responses

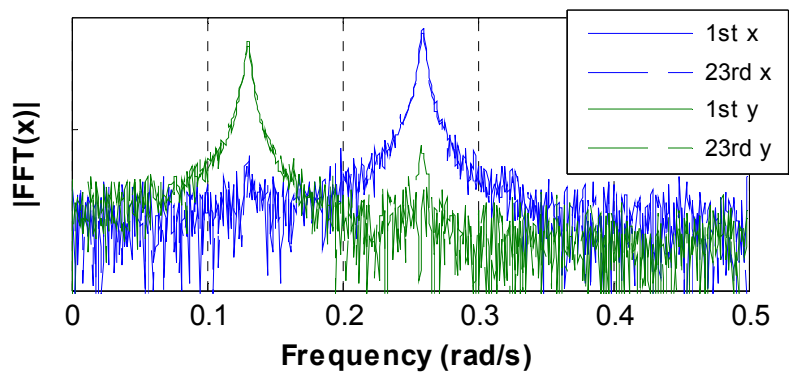

Figure 3: Responses of mass in $X$ and $Y$. (top) DFT of time response. (bottom) DFT of two of the lifted responses. 


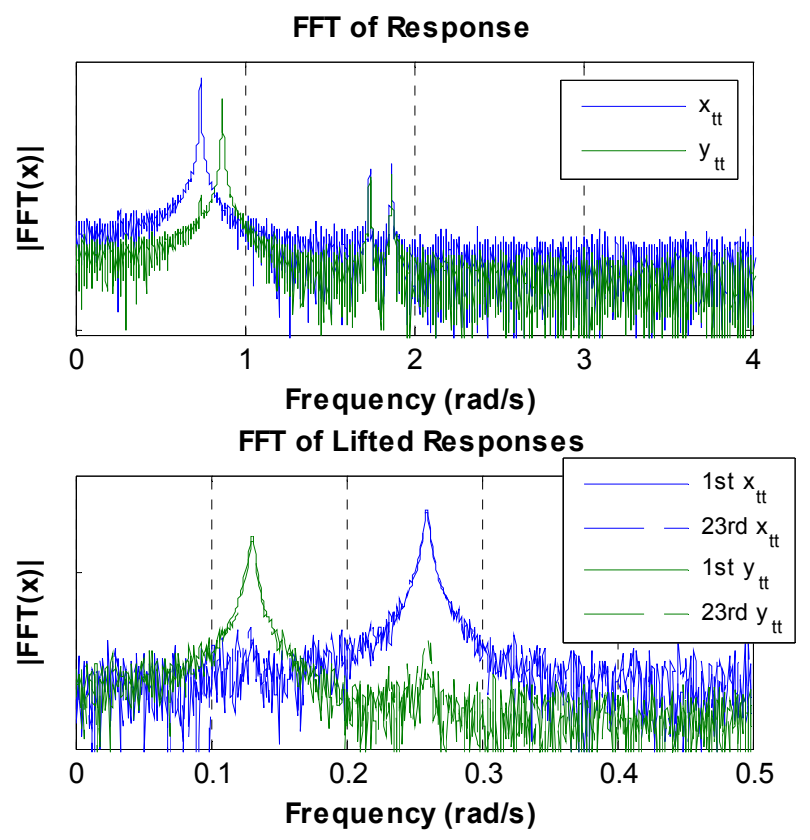

Figure 4: Responses of turntable $X_{t t}$ and $Y_{t t}$. (top) DFT of time response. (bottom) DFT of two of the lifted responses.

The response was also decomposed according to the lifting method, collecting the samples of the response for which the system is at the same angle (i.e. at $0+\theta$ and $180+\theta$ degrees). The response was sampled fifty times per half revolution of the shaft, so the lifting procedure creates in 50 sets of time responses for each output. The DFTs of these lifted responses was found, two of which are shown in the bottom pane of Figures 3 and 4 . The $1^{\text {st }}$ and $23^{\text {rd }}$ set of responses are shown corresponding to instants when the shaft was at $0^{\circ}$ or $180^{\circ}\left(1^{\text {st }}\right)$ and $79.2^{\circ}$ or $259.2^{\circ}\left(23^{\text {rd }}\right)$. Each FFT shows at most two resonant peaks, and the amplitude of the peaks is somewhat different in each of the responses shown, and indeed differed between all 50 lifted responses as expected.

The set of 200 lifted responses were processed using the Algorithm of Mode Isolation [51, 59-61], which considered all 200 responses simultaneously, automatically identifying both of the modes of the system. The respective residues for each response point-shaft angle combination were also identified by $\mathrm{AMI}$, and the algorithm verified that only two modes were present in the response by observing that the response was reduced to noise after removing these modal contributions from the data. This is illustrated in Figure 5, which shows a composite of the data, a composite of AMI's reconstruction of the data and a composite of the difference between the two. A composite is defined as the average magnitude of all of the lifted responses in the frequency domain [60]. The reconstruction agrees very well with the data, and the difference curve shows that the data appears to contain only noise after the contributions of these two modes are removed, confirming that only two modes are active. The identified Floquet exponents were:

$$
\begin{aligned}
& \left(\lambda_{\text {ident }}\right)_{1}=-0.002051+i^{*} 0.1302 \\
& \left(\lambda_{\text {ident }}\right)_{2}=-0.002036+i^{*} 0.2594
\end{aligned}
$$

The Floquet exponents were also computed numerically using adaptive Runge-Kutta integration to compute the STM over the fundamental period, and the following values were obtained, which agree quite closely with those shown above.

$$
\begin{aligned}
& \left(\lambda_{\text {analytical }}\right)_{1}=-0.002000+i^{*} 0.13034 \\
& \left(\lambda_{\text {analytical }}\right)_{2}=-0.002000+i^{*} 0.25939
\end{aligned}
$$




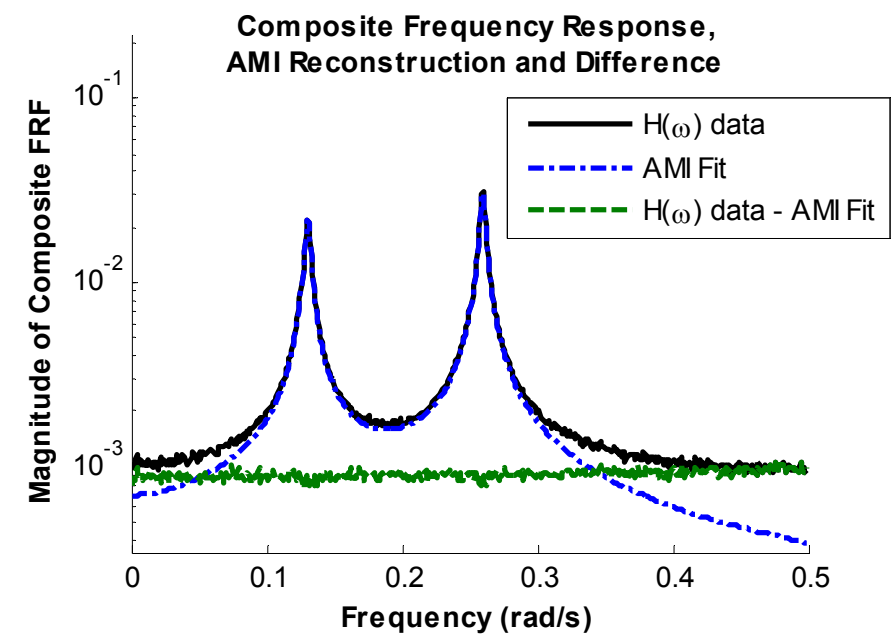

Figure 5: (solid, black) Composite of lifted responses, (dash-dot, blue) AMI reconstruction of composite and (dashed, green) composite of the difference between the two.

The real and imaginary parts of the residue for the first mode as a function of shaft angle are shown in Figure 6. The shaft angle is related to time by $\theta=\hat{\Omega} t$. The residues are predominantly constant, although they do vary slightly with shaft angle in a sinusoidal manner, and they are somewhat jagged, presumably due to the effect of the noise added to the responses.

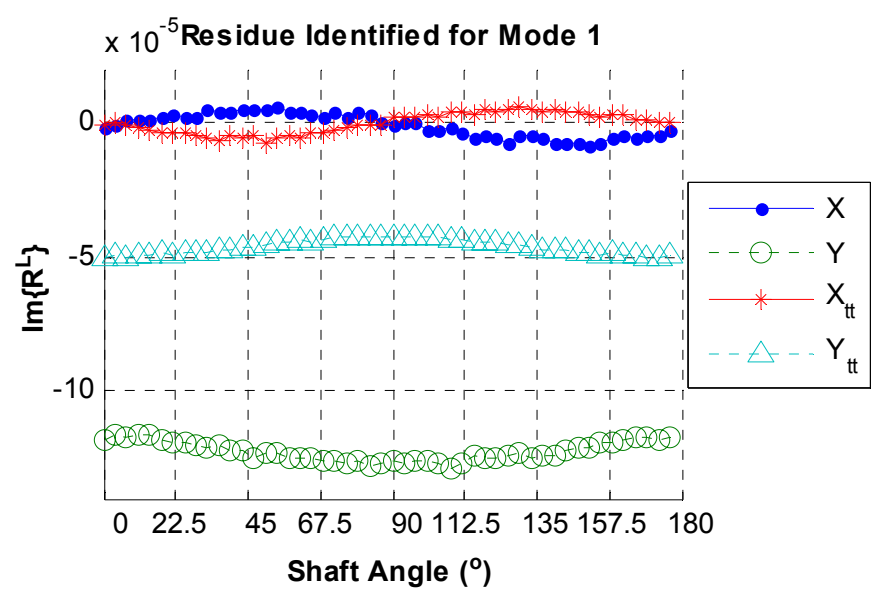

Figure 6: Residue vector of the first mode identified by AMI algorithm using lifting method. Only the imaginary part of the complex residue is shown.

\subsection{State Transition and System Matrices}

Two modes have been identified from the four responses for this system, so we can now use the methods developed in Sections 2.3 to find the STM and time-varying state matrix of the system for a subset of the responses that renders the system observable. The responses $X$ and $Y$ were used to do this, so $E$ in eq. (13) is a 2 by 4 matrix with $E_{11}=E_{22}=1$ and all other elements zero. The residues identified by AMI were converted to an FSE model by applying a DFT. The top pane in Figure 7 displays the absolute value of each of the coefficients of a DFT of the lifted residue for mode 1 , coordinate $X$. Only three terms stand out significantly above the noise for each residue. All of the other coefficients are smaller than the dominant ones, and appear to be on the order of the random fluctuations due to the artificially added noise, so they were discarded. The residue was reconstructed using the three retained Fourier coefficients, and its real and imaginary parts are shown as a function of shaft angle in the bottom pane. The reconstructed residue follows the general trend of the identified residue, but is much smoother. 

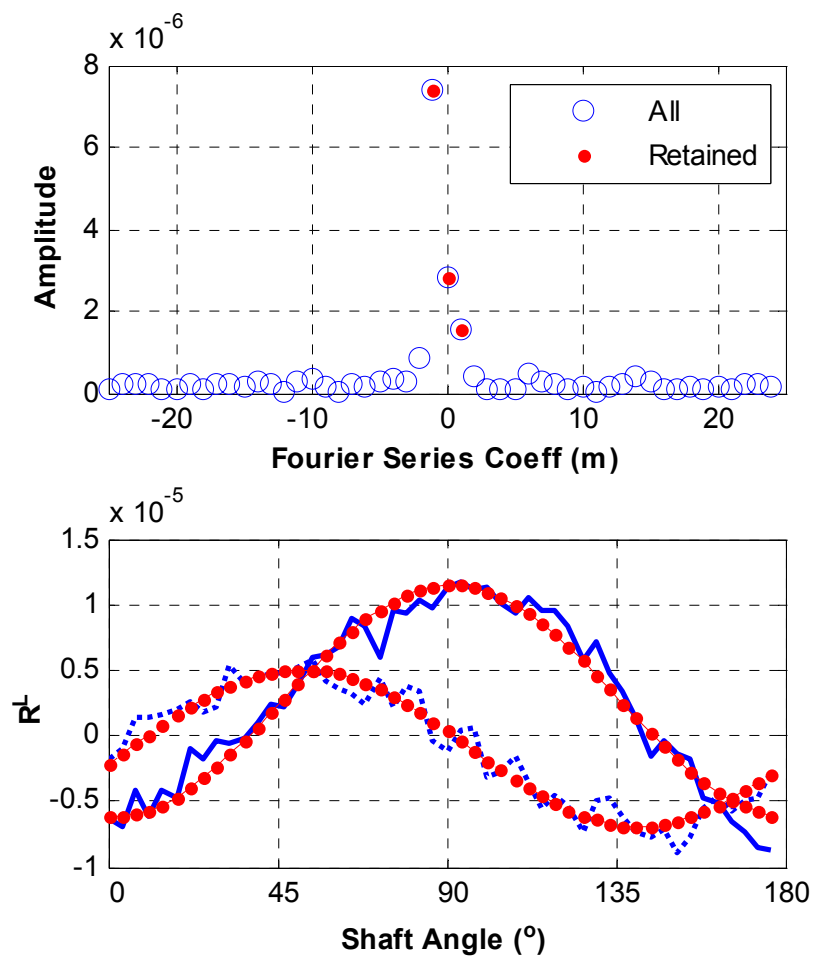

Figure 7: (top) DFT of $X$ component of the Residue Vector for Mode 1. Circles show all 50 DFT coefficients, points indicate the coefficients that were retained. (bottom) Plot of residue for mode 1.

Blue-lines show the real (solid) and imaginary (dashed) parts of the residue found by AMI, Redcircles show the reconstruction of the real and imaginary parts using only the three dominant coefficients in the expansion.

The retained Fourier coefficients were then used to reconstruct the state transition matrix using the procedure described in Section 2.3. The Fourier Series model for the residue matrices provided the $\left\{B_{x}\right\}_{r, m}$ coefficients required in eq. (18) to define the Floquet mode shapes $\{\psi(t)\}$, and the procedure described was used to find $\left\{L_{r}\left(t_{0}\right)\right\}$. The mode shapes, modal participation factors and corresponding Floquet exponents then define the state transition matrix via eq. (12). The derivative of the STM was also found, and the two were evaluated at fifty instants over the fundamental period of the LTP system (i.e. in the time interval $[0,2 \pi)$ ), and eq. (21) was then used to solve for the system matrix $A(t)$ at each time instant. 


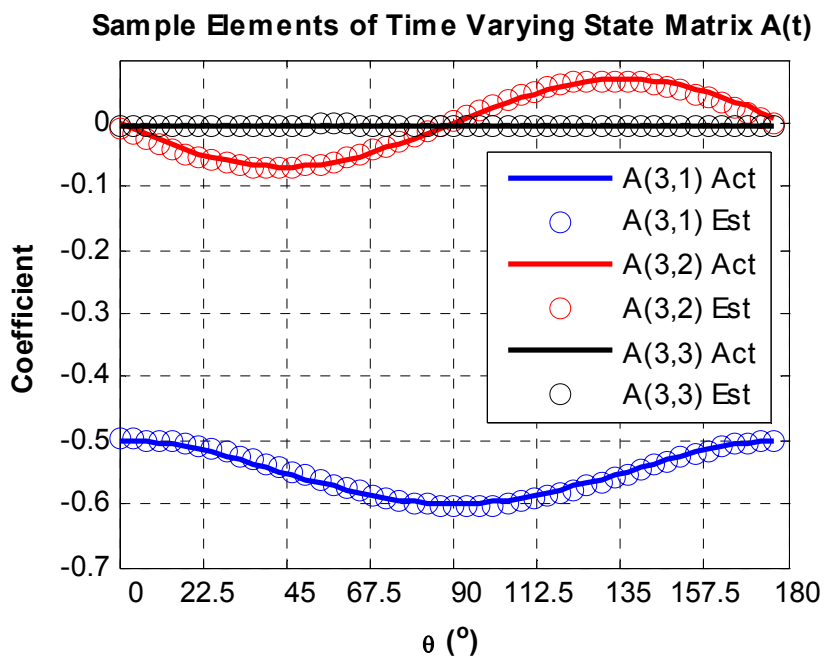

Figure 8: Sample coefficients of the time varying system matrix $A(t)$. (solid) Actual coefficient as a function of shaft angle, (circles) Coefficient estimated from noise contaminated response data. The curves overlay one another.

Figure 8 shows three of the coefficients of the state matrix that were estimated using this procedure, compared with the actual analytical coefficients. The $A(3,1) A(3,2)$ and $A(3,3)$ coefficients that are shown correspond, respectively, to the negatives of the $(1,1)$ and $(1,2)$ elements of the stiffness matrix and the $(1,1)$ element of the damping matrix for this system. As expected, at $\theta=0$ the $\mathrm{K}_{11}$ term is equal to $-0.5=-\left(k_{R x}{ }^{*} k_{F x}\right) /\left(k_{R x}+k_{F x}\right)$, which is the series stiffness of the system at that angle. The $\mathrm{K}_{12}$ term is zero for $\theta=0$ and increases to a maximum negative value at 45 degrees. The identified terms reveal that the stiffness matrix is sinusoidal, the dominant terms being the constant term, followed by terms that have one period per half revolution of the shaft. The on-diagonal stiffness in the $x$-direction is seen to vary moderately with shaft angle, fluctuating by about $20 \%$. The coupling stiffness between $x$ and $y$ is zero at 0,90 and 180 degrees and reaches a peak value of just under $20 \%$ of the on-diagonal $x$-direction stiffness at 45 and 135 degrees. The $(1,1)$ element of the damping matrix is much smaller than the stiffness terms and appears to be constant. It is significant to note that this system matrix was estimated without a priori knowledge of either its order nor of the form of its time-varying functions.

All three of the coefficients were accurately estimated using the proposed procedure. The maximum error in any coefficient of $A(t)$ over all shaft angles was 0.013 , corresponding to an off-diagonal term in the damping matrix. This corresponds to $1.3 \%$ error since the largest coefficient in $A(t)$ is one. However, the terms in the portion of $A(t)$ corresponding to the damping matrix are small, so the errors in those terms are almost $100 \%$ of their actual maximum values. The same procedure was also repeated with $X_{t t}$ and $Y_{t t}$ selected as the states. Once again three Fourier coefficients were sufficient to describe the identified residues. The $A(t)$ matrix estimated in that case was compared with that derived for the system, and the largest error found was $3.1 \%$ in the coupling stiffness between $X_{t t}$ and $Y_{t t}$.

\subsection{Discussion}

This paper has simulated an actual experiment by adding a considerable level of noise to the response of an LTP system. The noise masks all but a few of the peaks in the spectrum shown in the top pane in Figure 3. The difficulties associated with using the FSE method in this case have been discussed. When the lifting method is used, as in the example presented here, measurement noise makes the residues of the state transition matrix more jagged than they would otherwise be. While these residues can be used to accurately reconstruct the response, one does encounter difficulty when using them to reconstruct the time varying state matrix $A(t)$. The identified models must be differentiated in this process (see eq. (20)), which can amplify small high-frequency errors in the model.

This difficulty was overcome in this example by eliminating all of the Fourier coefficients that did not stand out above the noise floor. For this system, the $A(t)$ matrix was accurately estimated using either: 1) the three dominant Fourier coefficients (which created Figure 8), 2) the four dominant coefficients, or 3 ) all of the coefficients for which $-2 \leq \mathrm{m} \leq 2$. However, when all of the coefficients in the Fourier series expansion for which $-3 \leq \mathrm{m} \leq 3$ are retained, the maximum error in $A(t)$ increases to $6.2 \%$ 
and the estimate of the state matrix shows artificial waviness with three cycles per period. This suggests that the $\mathrm{m}= \pm 3$ coefficients are larger than they should be due to the noise, and that their effect is amplified when finding $A(t)$. A Fourier series expansion of the analytical residues was created to confirm this, revealing that the FSE coefficients of the analytical residues decrease as about $10^{-m}$, so the actual $m= \pm 3$ coefficients were well below the noise. If these coefficients were thought to be meaningful, one must design the experiment such that they stand out above the noise. Otherwise, they should be filtered from the response by truncating the Fourier series expansion so that they do not contribute adversely to the estimation of the time varying state matrix.

\section{Conclusions}

A methodology was presented by which one can identify a response model, the time varying state matrix and the state transition matrix of a linear time-periodic system from free response data. A series of pre- and post-processing steps were presented so that virtually any system identification algorithm for multi-output time-invariant systems can be used, and some guidelines were presented to aide in selecting an algorithm.

The proposed approach was demonstrated using synthesized, noise contaminated response data from a Jeffcott rotor on an anisotropic shaft, supported by an anisotropic foundation. The proposed lifting method was found to simplify the process of identifying the Floquet exponents of the LTP system and its model order; a standard system identification routine for LTI systems was able to automatically identify the modes in the response and the model order. This revealed that a subset of the measurement points was sufficient to describe the displacement state of the system. Two subsets of the measurement points were used to find the system's state transition matrix $\Phi\left(t, t_{0}\right)$ and its time-periodic system matrix $A(t)$, and in both cases each term in the system matrix was estimated within a few percent, even though the responses used to find them were contaminated with substantial noise. However, the procedure was shown to be sensitive to the Fourier Coefficients of the LTP modes corresponding to large $m$, so spurious coefficients had to be filtered from the response model to accurately reconstruct the state matrix. Failing to do so contaminated the reconstructed system matrix, although the response model does still faithfully reproduce that portion of the response that stands out above the measurement noise.

These methods could be applied to solve a variety of system identification problems. Efforts are already underway to use them to identify the mode shapes of structures from continuous-scan laser vibrometer measurements [43, 44]. They can also be applied to detect asymmetry (e.g. due to a crack) in the shaft of a rotor-bearing system [41], to experimentally derive models for complex rotating machines such as wind turbines, helicopters or tires, and to validate analysis models of LTP systems.

\section{References}

[1] Floquet, G., 1883, "Sur Les Equations Lineaires a Coefficients Periodiques," Ann. Sci. Ecole Norm. Sup., 12(Series 2), pp. 47-88.

[2] Peters, D. A., 1994, "Fast Floquet Theory and Trim for Multi-Bladed Rotorcraft," Journal of the American Helicopter Society, 39(4), pp. 82-89.

[3] Friedmann, P., Hammond, C. E., and Woo, T.-H., 1977, "Efficient Numerical Treatment of Periodic Systems with Application to Stability Problems," International Journal for Numerical Methods in Engineering, 11(pp. 1117-1136.

[4] Bir, G., and Stol, K., 1999, "Operating Modes of a Teetered-Rotor Wind Turbine," 17th International Modal Analysis Conference (IMAC XVII), Kissimmee, Florida,

[5] Montagnier, P., Spiteri, R. J., and Angeles, J., 2004, "The Control of Linear Time-Periodic Systems Using Floquet-Lyapunov Theory," International Journal of Control, 77(5), pp. 472-490.

[6] Oncescu, F., Lakis, A. A., and Ostiguy, G., 2001, "Investigation of the Stability and Steady State Response of Asymmetric Rotors, Using Finite Element Formulation," Journal of Sound and Vibration, 245(2), pp. 303-328.

[7] Fu, Y. M., and Zheng, Y. F., 2002, "Analysis of Non-Linear Dynamic Stability for a Rotating Shaft-Disk with a Transverse Crack," Journal of Sound and Vibration, 257(4), pp. 713-731.

[8] Flowers, G. T., Margithu, D. B., and Szasz, G., 1998, "The Application of Floquet Methods in the Analyses of Rotordynamic Systems," Journal of Sound and Vibration, 218(2), pp. 249-259.

[9] Desmidt, H. A., Wang, K. W., and Smith, E. C., 2002, "Coupled Torsion-Lateral Stability of a ShaftDisk System Driven through a Universal Joint," Journal of Applied Mechanics, 69(pp. 261-273. 
[10] Richards, J. A., 1983, Analysis of Periodically Time-Varying Systems, Springer-Verlag, Berlin, Heidelberg, New York,

[11] Rhoads, J. F., Shaw, S. W., Turner, K. L., Moehlis, J., Demartini, B. E., and Wenhua, Z., 2006, "Generalized Parametric Resonance in Electrostatically Actuated Microelectromechanical Oscillators," Journal of Sound and Vibration, 296(4-5), pp. 797-829.

[12] Rhoads, J. F., Miller, N. J., Shaw, S. W., and Feeny, B. F., 2007, "Mechanical Domain Parametric Amplification," ASME 2007 International Design Engineering Technical Conferences, IDETC/CIE 2007, Las Vegas, Nevada, USA, September 4-7.

[13] Sinha, S. C., and Pandiyan, R., 1994, "Analysis of Quasilinear Dynamical Systems with Periodic Coefficients Via Liapounov-Floquet Transformation," International Journal of Non-Linear Mechanics, 29(5), pp. 687-702.

[14] Bucher, I., and Shomer, O., 2005, "Detecting Asymmetry in Rotating Structures a Combined Acutation and Signal Processing Approach," 23rd International Modal Analysis Conference (IMAC XXIII), Orlando, Florida,

[15] Bendat, J. S., and Piersol, A. G., 1980, Engineering Applications of Correlation and Spectral Analysis, Wiley, New York.

[16] Hench, J. J., 1995, "A Technique for the Identification of Linear Periodic State-Space Models," International Journal of Control, 62(2), pp. 289-301.

[17] Verhaegen, M., and Xiaode, Y., 1995, "A Class of Subspace Model Identification Algorithms to Identify Periodically and Arbitrarily Time-Varying Systems," Automatica, 31(2), pp. 201-16.

[18] Felici, F., Van Wingerden, J. W., and Verhaegen, M., 2007, "Subspace Identification of MIMO LPV Systems Using a Periodic Scheduling Sequence," Automatica, 43(10), pp. 1684-97.

[19] Liu, K., 1997, "Identification of Linear Time-Varying Systems," Journal of Sound and Vibration, 206(4), pp. 487-505.

[20] Shi, Z. Y., Law, S. S., and Li, H. N., 2007, "Subspace-Based Identification of Linear Time-Varying System," AIAA Journal, 45(8), pp. 2042-50.

[21] Peters, D. A., and Wang, X., 1998, "Generalized Floquet Theory for Analysis of Numerical or Experimental Rotor Response Data," 24th European Rotorcraft Forum, Marseilles, France, September 15-17.

[22] Peters, D. A., and Su, A., 1990, "Effect of Hidden Dynamic States on Floquet Eigenvalues," Journal of the American Helicopter Society, 35(4), pp. 72-75.

[23] Fuehne, C. P., 2000, "Application of Generalized Floquet Theory to Numerical and Experimental Data," Ph.D. thesis, Washington University in St. Louis,, St. Louis, Missouri.

[24] Fuehne, C., 2000, "Application of Generalized Floquet Theory to Ground Resonance Data," 56th Annual National Forum of the American Helicopter Society, Virginia Beach, VA, May 1-4.

[25] Cauberghe, B., Guillaume, P., Verboven, P., Parloo, E., and Vanlanduit, S., 2004, "Frequency Domain Subspace Identification for Modal Analysis: How to Deal with Leakage and Transients?," 22nd International Modal Analysis Conference (IMAC XXII), Dearborn, Michigan,

[26] Pridham, B. A., and Wilson, J. C., 2004, "An Application Example Illustrating the Practical Issues of Subspace Identification," International Modal Analysis Conference (IMAC XXII), Dearborn, Michigan,

[27] Pintelon, R., Guillaume, P., Rolain, Y., Shoukens, J., and Van Hamme, H., 1994, "Parametric Identification of Transfer Functions in the Frequency Domain--a Survey," IEEE Transactions on Automatic Control, 39(11), pp. 2245-2260.

[28] Pintelon, R., and Schoukens, J., 2001, System Identification: A Frequency Domain Approach, IEEE Press, Piscataway, NJ.

[29] Schoukens, J., Pintelon, R., and Van Hamme, H., 1994, "Identification of Linear Dynamic Systems Using Piecewise Constant Excitations: Use, Misuse and Alternatives," Automatica, 30(7), pp. 11531169.

[30] Wereley, N. M., and Hall, S. R., 1991, "Linear Time Periodic Systems: Transfer Functions, Poles, Transmission Zeroes and Directional Properties," Proceedings of the 1991 American Control Conference, Boston, MA, USA,

[31] Siddiqui, A., 1999, "Identificaiton of the Harmonic Transfer Functions of a Helicopter Rotor," Ph.D. thesis, Massachusetts Institute of Technology,

[32] Sang Joon, S., Cesnik, C. E. S., and Hall, S. R., 2005, "System Identification Technique for Active Helicopter Rotors," Journal of Intelligent Material Systems and Structures, 16(11-12), pp. 1025-38. 
[33] Vanlanduit, S., Guillaume, P., and Schoukens, J., 2002, "Broadband Vibration Measurements Using a Continuously Scanning Laser Vibrometer," Measurement Science \&amp; Technology, 13(10), pp. 1574-82.

[34] Masri, S. F., and Caughey, T. K., 1979, "A Nonparametric Identification Technique for Nonlinear Dynamic Problems," Journal of Applied Mechanics, 46( ), pp. 433-447.

[35] Masri, S. F., Caffrey, J. P., Caughey, T. K., Smyth, A. W., and Chassiakos, A. G., 2004, "Identification of the State Equation in Complex Non-Linear Systems," International Journal of NonLinear Mechanics, 39(7), pp. 1111-1127.

[36] Kerschen, G., Worden, K., Vakakis, A. F., and Golinval, J.-C., 2006, "Past, Present and Future of Nonlinear System Identification in Structural Dynamics," Mechanical Systems and Signal Processing, 20(pp. 505-592.

[37] Adams, D. E., and Allemang, R. J., 1998, "Survey of Nonlinear Detection and Identification Techniques for Experimental Vibrations," International Seminar on Modal Analysis (ISMA 23), Leuven, Belgium,

[38] Arambel, P. O., and Tadmor, G., 1994, "Robust H Infinity Identification of Linear Periodic DiscreteTime Systems," International Journal of Robust and Nonlinear Control, 4(4), pp. 595-612.

[39] Luxemburg, L. A., 1990, "Frequency Analysis of Time-Varying Periodic Linear Systems by Using Modulo P Transforms and Its Applications to the Computer-Aided Analysis of Switched Networks," Circuits, Systems, and Signal Processing, 9(1), pp. 3-29.

[40] Liu, K., 1999, "Extension of Modal Analysis to Linear Time-Varying Systems," Journal of Sound and Vibration, 226(1), pp. 149-67.

[41] Allen, M., and Ginsberg, J. H., 2006, "Floquet Modal Analysis to Detect Cracks in a Rotating Shaft on Anisotropic Supports," 24th International Modal Analysis Conference (IMAC XXIV), St. Louis, MO, Jan. 30 - Feb. 2.

[42] Allen, M. S., 2007, "Floquet Experimental Modal Analysis for System Identification of Linear TimePeriodic Systems," ASME 2007 International Design Engineering Technical Conference, Las Vegas, NV, Sept. 4-7, 2007.

[43] Allen, M. S., and Sracic, M. W., 2008, "A Method for Generating Pseudo Single-Point Frfs from Continuous Scan Laser Vibrometer Measurements," 26th International Modal Analysis Conference (IMAC XXVI), Orlando, Florida, February, 2008.

[44] Allen, M. S., and Sracic, M. W., 2008, "Mass Normalized Mode Shapes Using Impact Excitation and Continuous-Scan Laser Doppler Vibrometry," 8th International Conference on Vibration Measurements by Laser Techniques, Ancona, Italy,

[45] D'angelo, H., 1970, Linear Time-Varying Systems: Analysis and Synthesis, Allyn and Bacon Series in Electrical Engineering, Allyn and Bacon, Boston.

[46] Meyer, R. A., and Burrus, C. S., 1975, "Unified Analysis of Multirate and Periodically Time-Varying Digital-Filters," IEEE Transactions on Circuits and Systems, CA22(3), pp. 162-168.

[47] Allemang, R. J., and Brown, D. L., 1998, "A Unified Matrix Polynomial Approach to Modal Identification," Journal of Sound and Vibration, 211(3), pp. 301-322.

[48] Rades, M., 1994, "A Comparison of Some Mode Indicator Functions," Mechanical Systems and Signal Processing, 8(4), pp. 459-474.

[49] Guillaume, P., Verboven, P., Vanlanduit, S., Van Der Auweraer, H., and Peeters, B., 2003, "A PolyReference Implementation of the Least-Squares Complex Frequency-Domain Estimator," International Modal Analysis Conference (IMAC XXI), Kissimmee, Florida,

[50] Van Der Auweraer, H., Guillaume, P., Verboven, P., and Vanlanduit, S., 2001, "Application of a FastStabilizing Frequency Domain Parameter Estimation Method," Journal of Dynamic Systems, Measurement, and Control, 123(pp. 651-658.

[51] Allen, M. S., 2005, "Global and Multi-Input-Multi-Output (Mimo) Extensions of the Algorithm of Mode Isolation (Ami)," Ph.D. thesis, Georgia Institute of Technology, Atlanta, Georgia.

[52] Allen, M. S., and Ginsberg, J. H., 2004, "Simo Extension of the Algorithm of Mode Isolation," IMAC 22 - XXII International Modal Analysis Conference, Dearborn, Michigan,

[53] Allen, M. S., and Ginsberg, J. H., 2005, "A Global, Single-Input-Multi-Output (Simo) Implementation of the Algorithm of Mode Isolation and Applications to Analytical and Experimental Data," Mechanical Systems and Signal Processing, Under Review(pp.

[54] Allen, M. S., and Ginsberg, J. H., 2005, "Global, Hybrid, Mimo Implementation of the Algorithm of Mode Isolation," 23rd International Modal Analysis Conference (IMAC XXIII), Orlando, Florida, 
[55] Ogata, K., 1994, Discrete-Time Control Systems, Prentice Hall, Upper Saddle River, New Jersey.

[56] Martarelli, M., 2001, "Exploiting the Laser Scanning Facility for Vibration Measurements," Ph.D. thesis, Imperial College, London.

[57] Stanbridge, A. B., Martarelli, M., and Ewins, D. J., 1999, "Scanning Laser Doppler Vibrometer Applied to Impact Modal Testing," 17th International Modal Analysis Conference - IMAC XVII, Kissimmee, FL, USA,

[58] Richardson, M. H., and Formenti, D. L., 1985, "Global Curve Fitting of Frequency Response Measurements Using the Rational Fraction Polynomial Method," 3rd International Modal Analysis Conference, Orlando, $\mathrm{Fl}$,

[59] Allen, M. S., and Ginsberg, J. H., 2004, "A Linear Least-Squares Version of the Algorithm of Mode Isolation for Identifying Modal Properties. Part li: Application and Assessment," Journal of the Acoustical Society of America (JASA), 116(2), pp. 908-915.

[60] Allen, M. S., and Ginsberg, J. H., 2006, "A Global, Single-Input-Multi-Output (Simo) Implementation of the Algorithm of Mode Isolation and Applications to Analytical and Experimental Data," Mechanical Systems and Signal Processing, 20(pp. 1090-1111.

[61] Ginsberg, J. H., and Allen, M. S., 2004, "A Linear Least-Squares Version of the Algorithm of Mode Isolation for Identifying Modal Properties. Part I: Conceptual Development," Journal of the Acoustical Society of America (JASA), 116(2), pp. 900-907. 